\title{
SU(2) Skyrme Vortices
}

\author{
Yu. P. Rybakov, A. M. Tarabay, and I. G. Chugunov \\ Department of Theoretical Physics, \\ Peoples' Friendship University of Russia, \\ 6, Miklukho-Maklay Str., 117198 Moscow, Russia. \\ E-mail: yrybakov@mx.pfu.edu.ru
}

\begin{abstract}
A regular method for constructing vortex-like solutions with cylindrical symmetry to the equations of the SU(2) Skyrme chiral model is proposed. A numerical estimate for the length density of mass is given.
\end{abstract}

The Skyrme model [1] proved its efficiency in modelling the structure of baryons [2] and nuclei [3] since the appearance of Witten's analysis of quark confinement problem $[4,5]$. The model considers pions as Goldstone bosons and uses the Lagrangian density

$$
\mathcal{L}=-\frac{1}{4 \lambda^{2}} \operatorname{Sp}\left(l_{\mu} l^{\mu}\right)+\frac{\varepsilon^{2}}{16} \operatorname{Sp}\left(\left[l_{\mu}, l_{\nu}\right]\left[l^{\mu}, l^{\nu}\right]\right),
$$

constructed from the chiral current $l_{\mu}=U^{+} \partial_{\mu} U, U \in S U(2)$. The energy in the model is estimated from below through the topological charge

$$
Q=-\frac{1}{24 \pi^{2}} \varepsilon^{i j k} \int d^{3} x \operatorname{Sp}\left(l_{i} l_{j} l_{k}\right)
$$

which takes integer values and can be interpreted as baryon number. In particular, the nucleon emerges as an absolutely stable state with the minimal energy in the first homotopic class $(Q=1)[6]$. Unfortunately the corresponding hedgehog configuration cannot be described analytically due to complexity of the nonlinear equations for the chiral field. The situation is aggravated for the higher homotopic classes in view of nonseparability of radial and angle variables. To overcome these difficulties we propose to approximate the configurations with higher charges by closed vortices. As 
a first step in this direction we consider in the present paper the simplest static vortex configuration given by the matrix

$$
U=\exp (i \tau \Theta(\rho)), \quad \tau=\left[\begin{array}{cc}
0 & e^{-i \varphi} \\
e^{i \varphi} & 0
\end{array}\right]
$$

with $\rho, \varphi$ being cylindrical coordinates. The configuration (2) appears to be equivariant under the group $G=T(z) \otimes \operatorname{diag}\left[S O(2)_{I} \otimes S O(2)_{S}\right]$ including the translation along the vortex and combined isotopic-space rotations around its axis. Usually the massive term proportional to $2-U-U^{+}$is added to the expression (1). Though its role being essential for the existence of regular solutions, we omit it in the present text, with the aim to illustrate the method and to simplify the formulae.

Substituting (2) in (1) amounts to the radial Lagrangian density for the chiral angle $\Theta(\rho)$ :

$$
\mathcal{L}=-\frac{1}{2 \lambda^{2}}\left(\Theta^{\prime 2}+\frac{\sin ^{2} \Theta}{\rho^{2}}\right)-\varepsilon^{2} \Theta^{\prime 2} \frac{\sin ^{2} \Theta}{\rho^{2}} .
$$

After the change of variable $\rho=\lambda \sqrt{2} e^{t},-\infty \leq t \leq+\infty$, we obtain the mechanical problem given by the action functional

$$
I[\Theta]=\int_{-\infty}^{+\infty} d t\left[\dot{\Theta}^{2}\left(1+\varepsilon^{2} e^{-2 t} \sin ^{2} \Theta\right)+\sin ^{2} \Theta\right]
$$

where the dimensionless parameter $\varepsilon^{2}$ is reserved for the technical purposes.

From (3) one derives the canonical momentum

$$
p=2 \dot{\Theta}\left(1+\varepsilon^{2} e^{-2 t} \sin ^{2} \Theta\right)=\partial_{\Theta} S
$$

and the Hamilton-Jacobi equation

$$
\partial_{t} S+\frac{1}{4}\left(\partial_{\Theta} S\right)^{2}\left(1+\varepsilon^{2} e^{-2 t} \sin ^{2} \Theta\right)^{-1}-\sin ^{2} \Theta=0 .
$$

Now we search for the solution to the Eq.(5) as a formal series

$$
S(t, \Theta)=\sum_{n=0}^{\infty} \varepsilon^{2 n} S_{n}(t, \Theta) .
$$

Inserting (6) into (5) we get the recurrence relation

$$
\frac{1}{4} \sum_{l=0}^{n} \partial_{\Theta} S_{l} \partial_{\Theta} S_{n-l}+\partial_{t} S_{n}+\partial_{t} S_{n-1} e^{-2 t} \sin ^{2} \Theta=\delta_{n 0} \sin ^{2} \Theta+\delta_{n 1} e^{-2 t} \sin ^{4} \Theta
$$


with the evident private solution for $n=0$ :

$$
S_{0}=W_{0}(\Theta)=2 \cos \Theta
$$

In order to satisfy (7) we put for $n>0$

$$
S_{n}(t, \Theta)=e^{-2 n t} W_{n}(\Theta) .
$$

From (7), (8) and (9) we derive for $n=1$ the equation

$$
\sin \Theta W_{1}^{\prime}+2 W_{1}+\sin ^{4} \Theta=0
$$

with the two different solutions (two branches) corresponding to the ranges $\pi / 2 \leq \Theta \leq \pi$ and $0 \leq \Theta \leq \pi / 2$ respectively:

$$
\begin{gathered}
W_{1}^{+}(\Theta)=\frac{1}{3}(1+\cos \Theta)^{2}\left(\frac{4}{1-\cos \Theta}+3-\cos \Theta\right), \\
W_{1}^{-}(\Theta)=-\frac{1}{3}(1-\cos \Theta) \sin ^{2} \Theta .
\end{gathered}
$$

For $n>1$ we deduce from (7), (8) and (9) the equation

$$
\sin \Theta W_{n}^{\prime}+2 n W_{n}=\frac{1}{4} \sum_{l=1}^{n-1} W_{l}^{\prime} W_{n-l}^{\prime}-2(n-1) \sin ^{2} \Theta W_{n-1}
$$

with the solution

$$
\begin{aligned}
& W_{n}^{ \pm}(\Theta)=\operatorname{tg}^{-2 n}(\Theta / 2) \int_{a_{ \pm}}^{\Theta} \frac{d \Theta}{\sin \Theta} \operatorname{tg}^{2 n}(\Theta / 2) \times \\
& \quad\left[\frac{1}{4} \sum_{l=1}^{n-1} W_{l}^{\prime} W_{n-l}^{\prime}-2(n-1) \sin ^{2} \Theta W_{n-1}\right],
\end{aligned}
$$

where $a_{+}=\pi, a_{-}=0$. Formulae (10), (11) and (12) determine the recurrent procedure for constructing the solution to the Hamilton-Jacobi equation (5). Putting this solution to the r.h.s. of the equation (4), one can find the canonical momentum $p$ for the two branches of solution:

$$
p=2 \dot{\Theta}\left(1+\varepsilon^{2} e^{-2 t} \sin ^{2} \Theta\right)=\sum_{n=0}^{\infty} \varepsilon^{2 n} e^{-2 n t} W_{n}^{\prime}(\Theta) .
$$

For matching these branches at the point $\Theta=\pi / 2, t=t_{0}$ one deduces from (13) the algebraic equation

$$
\sum_{n=1}^{\infty} \varepsilon^{2 n} e^{-2 n t_{0}}\left[W_{n}^{+^{\prime}}(\pi / 2)-W_{n}^{-^{\prime}}(\pi / 2)\right]=0 .
$$


In particular, within the scope of $n=2$ approximation one gets from (14) the effective development parameter

$$
\xi=\frac{1}{3} \varepsilon^{2} e^{-2 t_{0}}=2(16 \ln 2-37 / 15)^{-1} \approx 0,232
$$

that permits to calculate the mass of the vortex (its length density):

$$
\begin{aligned}
& M=\frac{\pi}{\lambda^{2}} I=\frac{\pi}{\lambda^{2}}\left[S^{-}(+\infty, 0)-S^{-}\left(t_{0}, \pi / 2\right)+S^{+}\left(t_{0}, \pi / 2\right)-S^{+}(-\infty, \pi)\right]= \\
& \frac{4 \pi}{\lambda^{2}}\left[1+2 \xi+\xi^{2}(41 / 15-8 \ln 2)+O\left(\xi^{3}\right)\right] \approx \frac{4 \pi}{\lambda^{2}} 1,31 .
\end{aligned}
$$

In conclusion we notice that the function $\Theta(t)$, defining the radial distribution of matter inside the vortex, can be found from Eq.(13) which is represented in the integral form:

$$
\begin{aligned}
& \operatorname{tg} \frac{\Theta}{2}=e^{t_{0}-t} \exp \left\{\int_{t_{0}}^{t} d t\left(1+\varepsilon^{2} e^{-2 t} \sin ^{2} \Theta\right)^{-1} \times\right. \\
& \left.\left(\varepsilon^{2} e^{-2 t} \sin ^{2} \Theta+\frac{1}{2} \sum_{n=1}^{\infty} \varepsilon^{2 n} e^{-2 n t} \frac{W_{n}^{\prime}}{\sin \Theta}\right)\right\} .
\end{aligned}
$$

\section{References}

[1] Skyrme T.H.R. A Unified Field Theory of Mesons and Baryons // Nucl. Phys. 1962. 31. N4. P.556-569.

[2] Adkins G.S, Nappi. Ch.R., Witten E. Static Properties of Nucleons in the Skyrme Model // Nucl. Phys., ser.B, 1983. 228. N4. P.552-556.

[3] Nikolayeva R. M., Nikolayev V. A., Tkachov O.G. Nucleus-like States in the SU(2) Skyrme Model //Physics of Elementary Particles and Atomic Nuclei. 1992. 23. N2. P. 542-571.

[4] Witten E. Global Aspects of Current Algebra // Nucl. Phys., ser.B, 1983. 223. N3. P. 422-432.

[5] Witten E. Current Algebra, Baryons and Quark Confinement // Nucl. Phys., ser.B, 1983. 223. N3. P. 433-444.

[6] Rybakov Yu. P. Stability of Many-Dimensional Solitons in Chiral Models and Gravitation // VINITI series "Classical Field Theory and Gravitational Theory". V.2. Gravitation and Cosmology. Moscow: VINITI, 1991. P.56-111. 